\title{
Predictors of Highly Prevalent Brain Ischemia in Intracerebral Hemorrhage
}

\author{
Ravi S. Menon, MD, ${ }^{1}$ Richard E. Burgess, MD, PhD, ${ }^{1}$ Jeffrey J. Wing, MPH, ${ }^{2}$ \\ M. Christopher Gibbons, MD, MPH, ${ }^{3}$ Nawar M. Shara, PhD, ${ }^{4}$ Stephen Fernandez, MPH, ${ }^{4}$ \\ Annapurni Jayam-Trouth, MD, ${ }^{5}$ Laura German, BS, ${ }^{1}$ lan Sobotka, BS, \\ Dorothy Edwards, $\mathrm{PhD}^{6}$ and Chelsea S. Kidwell, MD ${ }^{1}$
}

\begin{abstract}
Objective: This study was undertaken to determine the prevalence, characteristics, risk factors, and temporal profile of concurrent ischemic lesions in patients with acute primary intracerebral hemorrhage (ICH).

Methods: Patients were recruited within a prospective, longitudinal, magnetic resonance imaging (MRI)-based study of primary ICH. Clinical, demographic, and MRI data were collected on all subjects at baseline and 1 month.

Results: Of the 138 patients enrolled, mean age was 59 years, 54\% were male, 73\% were black, and $84 \%$ had a history of hypertension. At baseline, ischemic lesions on diffusion-weighted imaging (DWI) were found in 35\% of patients. At 1 month, lesions were present in $27 \%$, and of these lesions, $83 \%$ were new and not present at baseline. ICH volume ( $p=$ 0.025), intraventricular hemorrhage $(p=0.019)$, presence of microbleeds $(p=0.024)$, and large, early reductions in mean arterial pressure $(p=0.003)$ were independent predictors of baseline DWI lesions. A multivariate logistical model predicting the presence of 1-month DWI lesions included history of any prior stroke $(p=0.012)$, presence of 1 or more microbleeds $(p=0.04)$, black race $(p=0.641)$, and presence of a DWI lesion at baseline $(p=0.007)$.

Interpretation: This study demonstrates that $>1 / 3$ of patients with primary $\mathrm{ICH}$ have active cerebral ischemia at baseline remote from the index hematoma, and $1 / 4$ of patients experience ongoing, acute ischemic events at 1 month. Multivariate analyses implicate blood pressure reductions in the setting of an active vasculopathy as a potential underlying mechanism. Further studies are needed to determine the impact of these lesions on outcome and optimal management strategies to arrest vascular damage.
\end{abstract}

ANN NEUROL 2012;71:199-205

ntracerebral hemorrhage ( $\mathrm{ICH})$ is a devastating disorder associated with substantial morbidity and mortality. ${ }^{1}$ The often dismal outcome for patients with $\mathrm{ICH}$ highlights the need to better understand the underlying complex pathophysiology of this disease process, which in turn can assist in accurate determination of future stroke risk, long-term outcome assessment, and perhaps most importantly, development of treatment interventions.

Advanced magnetic resonance imaging (MRI) techniques have become increasingly valuable in characterizing and refining our understanding of $\mathrm{ICH}$ pathophysiology. ${ }^{2}$ In addition to demonstrating acute hemorrhage, MRI can identify leukoaraiosis, chronic hematomas, and microbleeds, thus providing insight into the etiology and severity of the underlying disease process. Several recent
MRI studies have identified a high prevalence of topographically distant ischemic lesions identified on diffusionweighted imaging (DWI) in the setting of primary $\mathrm{ICH}^{3-5}$ However, the underlying mechanism, time course, and significance of these lesions is poorly understood.

This study seeks to determine the prevalence, characteristics, risk factors, and temporal evolution of silent ischemic lesions in a predominantly black, hypertensive population of patients with primary $\mathrm{ICH}$.

\section{Patients and Methods}

The current analysis was undertaken as part of an ongoing, prospective, longitudinal MRI study of racial differences in primary ICH (DiffErenCes in the Imaging of Primary Hemorrhage based on Ethnicity or Race: DECIPHER). Since 2007,

View this article online at wileyonlinelibrary.com. DOI: 10.1002/ana.22668

Received Jul 11, 2011, and in revised form Oct 26, 2011. Accepted for publication Nov 4, 2011.

Address correspondence to Dr Menon, Department of Neurology, Georgetown University, 4000 Reservoir Road, Suite 150, Washington, DC 20007. E-mail: rsm53@georgetown.edu

From the ${ }^{1}$ Department of Neurology and Stroke Center, Georgetown University, Washington, DC; ${ }^{2}$ Department of Biostatistics, University of Michigan, Ann Arbor, Ml; ${ }^{3}$ Johns Hopkins University Urban Health Institute, Baltimore, MD; ${ }^{4}$ Medstar Health Research Institute, Hyattsville, MD; ${ }^{5}$ Department of Neurology, Howard University, Washington, DC; and ${ }^{6}$ Departments of Kinesiology and Neurology, University of Wisconsin, Madison, WI. 
consecutive patients have been recruited from the inpatient services of 5 Washington, DC metropolitan area hospitals. Inclusion criteria are: age $\geq 18$ years, diagnosis of primary ICH (not limited to first $\mathrm{ICH}$ ), and brain MRI within 1 month of symptom onset. Exclusion criteria are: contraindication to MRI, pregnancy, central nervous system (CNS) tumor/active inflammatory process, CNS arteriovenous malformation/aneurysm, CNS trauma within prior 2 weeks, craniotomy, or international normalized ratio $>3$. Baseline demographic information, medical history, medications, neurologic assessments, and laboratory data are collected on all patients. Blood pressure (BP) measurements are collected for the following time points: admission, highest BP prior to MRI, lowest BP prior to MRI, and 1 month. Differences in mean arterial pressures (delta MAPs) are calculated for the various time intervals. Results of cardiac evaluations including electrocardiograms and echocardiograms are reviewed in combination with cardiac history, such that each patient is categorized as having a high-, low-, or no-risk cardioembolic source.

As part of the DECIPHER protocol, MRIs and outcome assessments are performed at admission (baseline), 1 month, 1 year, and 3 years. Baseline MRIs are acquired on either $1.5 \mathrm{~T}$ or 3.0T magnetic resonance (MR) scanners. All follow-up MR scans are preferentially acquired on a $3.0 \mathrm{~T}$ scanner. A standardized protocol is employed for all time points that includes DWI and apparent diffusion coefficient (ADC), fluid attenuated inversion recovery, and gradient recalled echo (GRE) sequences.

The DECIPHER study is being performed with approval of the institutional review boards (IRBs) of the admitting hospitals and Georgetown University, which serves as the IRB of record.

\section{Imaging Analyses}

For the current analysis, 3 investigators (R.S.M., R.E.B., C.S.K.) performed the imaging evaluations. An initial series of 15 cases were analyzed as consensus reads to establish inter-rater reliability. All imaging analyses were performed using Mango (Multi-Image Analysis GUI, http://ric.uthscsa.edu/mango/). GRE sequences were evaluated for the following: hematoma volume (using a semiautomated segmentation tool) and location; number and location of microbleeds and chronic hematomas; and presence of intraventricular hemorrhage (IVH). Microbleeds were defined as rounded foci of hypointense signal on GRE sequences within the brain parenchyma. ${ }^{6}$

DWI/ADC sequences were evaluated to characterize the presence, number, location, volume, and acuity of ischemic lesions topographically remote from the hematoma. DWI hyperintensities immediately adjacent to the hematoma were not included in this analysis. A comparison of equivalent axial slices from the 2 time points was performed for each DWI lesion visualized on the 1 month scan to determine (1) if it was new (or persistent from the baseline time point); and (2) if new, whether it was acute (low ADC value), or subacute (normal or elevated ADC value). Lateralized assessment of the topography and severity of leukoaraiosis was assessed using the Fazekas scale ${ }^{7}$ for periventricular (PV) and deep white matter (DWM) regions. A sum score of white matter disease was calculated as the sum of the PV and DWM scores.

\section{Statistical Methods}

Differences in dichotomous variables were analyzed using chisquare analysis or the Fisher exact test. Student $t$ test or the Wilcoxon rank sum test was used to analyze differences in the mean or median of continuous variables between groups. Variables that indicated a univariate relationship with lesion presence $(p<0.1)$ and were not strongly correlated with each other were considered for multivariate logistic modeling. All statistical analyses were performed using SAS version 9.2 (SAS Institute, Cary, NC).

\section{Results}

During the study time frame, 138 of 452 (31\%) patients meeting study eligibility criteria were enrolled. Of these, 119 subjects had a baseline MRI, 113 subjects had a 1-month MRI, and 94 subjects had imaging at both baseline and 1 -month time points. For the overall cohort, mean age was 59 years, $73 \%$ of patients were black, $54 \%$ were male, $84 \%$ had a history of hypertension, and median National Institutes of Health Stroke Scale ${ }^{8}$ score was 5 (mean, 9). On imaging, mean hematoma volume was $25 \mathrm{ml}$ (median, $14 \mathrm{ml}$; range, $0.4-136.4 \mathrm{ml}$ ), and $74 \%$ of hematomas originated in deep structures. The underlying etiology of the hemorrhage was determined to be hypertension in $62 \%$ of patients, cerebral amyloid angiopathy in $1.5 \%$, and unknown (no risk factors) in $3.6 \%$, with the remainder due to hypertension plus $\geq 1$ additional risk factors. Median time was 2 days to baseline MRI and 35 days to 1-month MRI.

At the baseline time point, $35 \%$ of patients had $\geq 1$ DWI lesions (median, 2; range, 1-67), for a total of 227 lesions in 42 of 119 patients. At the 1-month time point, $27 \%$ of patients had $\geq 1$ DWI lesions (median, 2; range, 1-7), for a total of 67 lesions in 30 of 113 patients. Lesions were generally small at both time points; median volume was $0.5 \mathrm{ml}$ (range, $0.04-16 \mathrm{ml}$ ) for baseline lesions and $0.4 \mathrm{ml}$ (range, $0.01-14 \mathrm{ml}$ ) for 1-month lesions. Figure 1 provides an example of a patient with multiple baseline DWI lesions, and Figure 2 shows an example of a patient with a new acute lesion visualized at 1 month and not present at baseline.

To clarify the evolution, acuity, and time course of the ischemic lesions, an analysis was performed on the subset of patients with MRIs at both baseline and 1-month time points. In this subset, there were 158 lesions at baseline, and of these, $78 \%$ were identified as acute (low $\mathrm{ADC}$ ) and $22 \%$ as subacute (iso- or hyperintense ADC). At 1 month, there were a total of 40 lesions; of these, $58 \%$ were acute and $42 \%$ were subacute. In total, $87 \%$ of patients with lesions at 1 month had $\geq 1$ new DWI lesions compared to baseline, and $83 \%$ of all lesions at 1 month were new compared to baseline. Of these new lesions, $64 \%$ were acute and $36 \%$ were subacute.

Additional analyses were performed to further characterize the topography and laterality of the DWI lesions. 


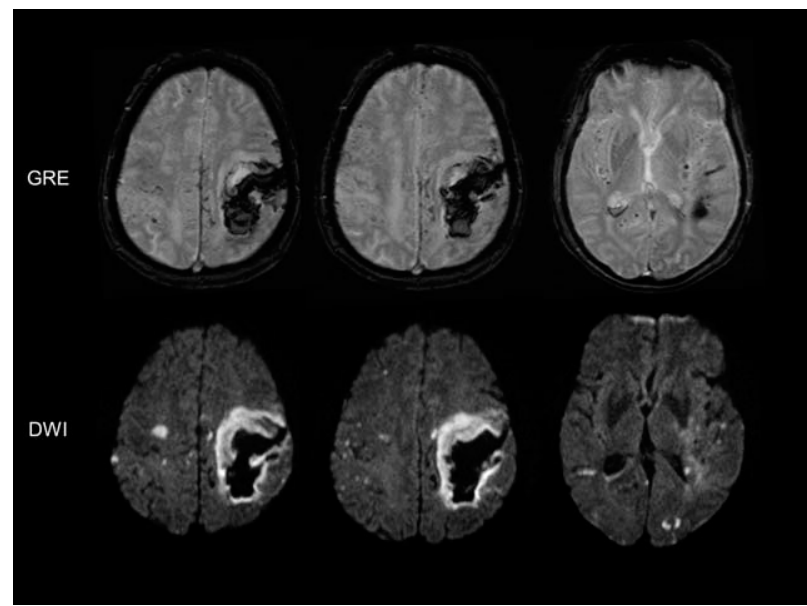

FIGURE 1: Serial axial magnetic resonance images of symptomatic primary intracerebral hemorrhage $(\mathrm{ICH})$ in a 69-year-old hypertensive white female. Top row shows the gradient recalled echo (GRE) sequence demonstrating a left parietofrontal primary $\mathrm{ICH}$. Bottom row shows scattered hyperintensities on diffusion-weighted imaging (DWI) at the baseline time point remote from the primary $\mathrm{ICH}$. A complete workup for embolic stroke was negative.

At baseline, $44 \%$ of the DWI lesions were located in lobar locations, whereas at 1 month, only $13 \%$ were lobar. At both the baseline and 1-month time points, approximately $1 / 3$ of patients had DWI lesions ipsilateral to the primary hematoma, $1 / 3$ had contralateral lesions, and $1 / 3$ had bilateral lesions (Fig 3).

Of the 138 patients, 49 (35.5\%) patients had either a transthoracic or transesophageal echocardiogram performed. Only 9 patients were deemed to have a high-risk

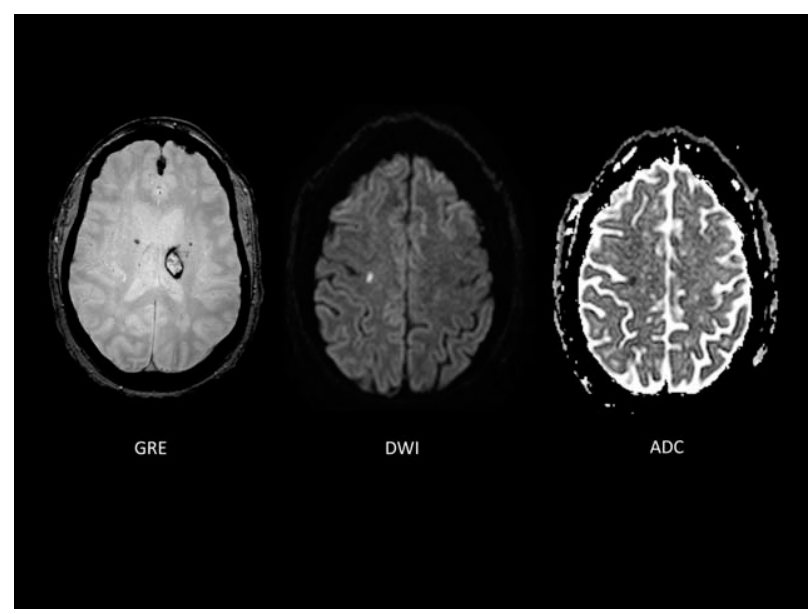

FIGURE 2: Axial gradient recalled echo (GRE), diffusionweighted imaging (DWI), and apparent diffusion coefficient (ADC) images from the 1-month time point in a 59 -year-old African American female. GRE sequence demonstrates a subacute left periventricular hematoma and several deep microbleeds. DWI image from a higher slice shows a hyperintense lesion in the right frontal lobe, and corresponding ADC map shows hypointensity indicative of an acute event. The lesion was not present on baseline imaging.

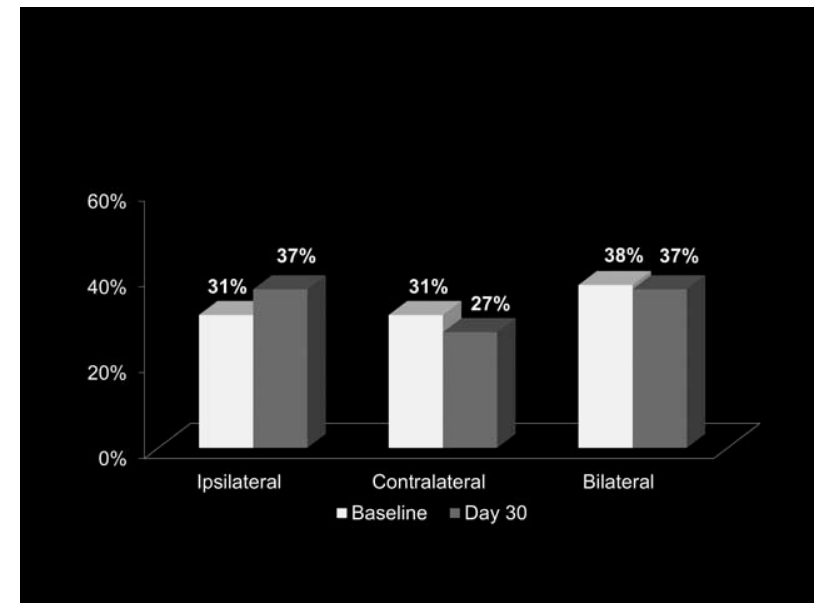

FIGURE 3: Frequency of diffusion-weighted imaging ischemic lesion laterality for the baseline and 1-month time points.

cardioembolic source, and this did not differ significantly between those with DWI lesions versus without $(p=$ 0.179). Thirty-two patients (23\%) had diagnostic cerebral angiograms performed, with no difference in the frequency of angiography between those with DWI lesions versus those without (baseline, $p=0.134 ; 1$ month, $p=0.325$ ).

Univariate analyses were performed for baseline and 1 month, respectively, to identify differences in characteristics for patients with $\geq 1$ ischemic DWI lesions versus those without. Table 1 shows the results for the baseline time point. In contrast to patients without baseline lesions, patients with lesions were significantly more likely to have a lobar ICH $(p=0.027)$, larger ICH volume $(p=0.033)$, microbleeds $(p=0.015)$, IVH $(p=0.002)$, and greater mean delta MAP $(p<0.001)$, and were more likely to be treated with antihypertensive agents during the acute hospital stay $(p=0.031)$. In multivariate logistical modeling, hematoma volume, IVH, microbleeds, and delta MAP remained independent predictors of the presence of baseline DWI lesions (Table 2).

Univariate analysis for the 1-month time point (Table 3) demonstrated that patients with DWI lesions had a greater likelihood of more severe leukoaraiosis $(p=0.004)$, microbleeds $(p=0.004)$, chronic hematomas $(p=0.019)$, DWI lesions at baseline $(p=0.001)$, and greater mean delta MAP $(p<0.001)$. There was a trend for DWI lesions to occur with greater frequency in black patients $(p=0.056)$ and in patients with prior stroke history $(p=0.056)$. A multivariate logistic regression model predicting the presence or absence of 1-month DWI lesions was developed that included black race, history of any prior stroke, presence of $\geq 1$ microbleeds, and presence of a DWI lesion at baseline (Table 4). As mean delta MAP was significantly correlated with baseline DWI lesions, it was not incorporated into the 1-month model. In this study with a primary focus on racial disparities in 


\begin{tabular}{|c|c|c|c|c|}
\hline Characteristic & $\begin{array}{l}\text { All Subjects, } \\
\mathrm{n}=119\end{array}$ & $\begin{array}{l}\text { DWI Positive, } \\
\mathrm{n}=42\end{array}$ & $\begin{array}{l}\text { DWI Negative, } \\
\mathbf{n}=77\end{array}$ & $p$ \\
\hline Age, mean yr (SD) & $59.8(13.1)$ & $60.9(10.6)$ & $59.1(14.3)$ & 0.445 \\
\hline Black race, No. [\%] & $87[73.1]$ & $32[76.2]$ & $54[71.1]$ & 0.575 \\
\hline Male gender, No. [\%] & $65[54.6]$ & 26 [61.9] & $38[50.0]$ & 0.239 \\
\hline Hypertension, No. [\%] & $100[84.0]$ & $36[85.7]$ & $63[82.9]$ & 0.573 \\
\hline Delta MAP, mean mmHg (SD) & $40.2(24.1)$ & $50.8(25.0)$ & $34.4(21.7)$ & $<0.001$ \\
\hline $\begin{array}{l}\text { Acute in-hospital antihypertensive } \\
\text { treatment, No. [\%] }{ }^{\mathbf{a}}\end{array}$ & $101[88.6]$ & $39[97.5]$ & $62[83.8]$ & 0.031 \\
\hline NIHSS, median \{range\} & $5\{0-40\}$ & $7.5\{0-40\}$ & $4\{0-35\}$ & 0.136 \\
\hline Prior stroke, any type, No. [\%] & $33[27.7]$ & $15[35.7]$ & $18[23.7]$ & 0.151 \\
\hline High-risk CES, No. [\%] & $9[7.6]$ & $4[9.5]$ & $5[6.5]$ & 0.179 \\
\hline Lobar ICH, No. [\%] & $31[26.1]$ & $16[38.1]$ & $15[19.7]$ & 0.027 \\
\hline Pre-ICH antithrombotic use, No. [\%] ${ }^{\mathbf{b}}$ & $31[26.1]$ & $11[26.2]$ & $20[27]$ & 0.922 \\
\hline ICH volume, median ml $\{\mathrm{IQR}\}$ & $13.9\{0.4-136.4\}$ & $25.1\{0.6-109.9\}$ & $12.6\{0.4-136.4\}$ & 0.033 \\
\hline IVH, No. [\%] & $48[40.3]$ & $25[59.5]$ & $23[30.3]$ & 0.002 \\
\hline Microbleeds, No. [\%] & 69 [59.0] & $31[73.8]$ & $38[51.4]$ & 0.015 \\
\hline Chronic hematomas, No. [\%] & $36[30.5]$ & $15[35.7]$ & $21[28.0]$ & 0.361 \\
\hline WMD score, median \{range\} & $6\{0-12\}$ & $6\{2-12\}$ & $6\{0-12\}$ & 0.105 \\
\hline
\end{tabular}

a sample of predominantly black $\mathrm{ICH}$ patients, black race was integrated into the model, given the significant associations with microbleeds and vascular risk factors.

\section{Discussion}

\section{Summary of Findings}

This prospective, longitudinal MRI-based study of primary ICH not only confirms prior studies reporting a high frequency of ischemic lesions (topographically remote from the hematoma) in the acute phase of primary $\mathrm{ICH}$, but also extends these findings to provide important new insights into the dynamic nature and underlying etiology of these lesions. Our analysis identifies active regions of remote ischemia at baseline in $>1 / 3$ of patients; follow-up imaging at 1 month demonstrates ischemic lesions in $>1 / 4$. Notably, $83 \%$ of 1 -month lesions were new and not present on baseline imaging, a finding indicative of an ongoing and active pathologic process. Independent predictors of DWI lesions at baseline

\section{TABLE 2: Multivariate Logistic Model for Presence of Baseline DWI Lesions}

\begin{tabular}{lcccc} 
Factor & Odds Ratio & \multicolumn{2}{c}{$\mathbf{9 5 \%}$ CI } & $\boldsymbol{p}$ \\
Hematoma volume, ml & 1.02 & 1.00 & 1.04 & 0.025 \\
Baseline IVH & 3.00 & 1.20 & 7.48 & 0.019 \\
Baseline microbleeds & 2.94 & 1.16 & 7.49 & 0.024 \\
Delta MAP, mmHg & 1.03 & 1.01 & 1.05 & 0.003 \\
CI = confidence interval; IVH = intraventricular hemorrhage; MAP = mean arterial pressure. &
\end{tabular}




\begin{tabular}{|c|c|c|c|c|}
\hline Characteristic & $\begin{array}{l}\text { All Subjects, } \\
\mathrm{n}=113\end{array}$ & $\begin{array}{l}\text { DWI Positive, } \\
\mathbf{n}=\mathbf{3 0}\end{array}$ & $\begin{array}{l}\text { DWI Negative, } \\
\mathrm{n}=\mathbf{8 3}\end{array}$ & $p$ \\
\hline Age, mean (SD) & $58.3(12.4)$ & $58.8(10.0)$ & $58.0(13.3)$ & 0.751 \\
\hline Black race, No. [\%] & $83[73.5]$ & $26[86.7]$ & $57[68.7]$ & 0.056 \\
\hline Male gender, No. [\%] & $62[54.9]$ & $19[63.3]$ & $43[51.8]$ & 0.277 \\
\hline Hypertension, No. [\%] & $91[80.5]$ & $27[90.0]$ & $64[77.1]$ & 0.28 \\
\hline Delta MAP, mean mmHg (SD) & $40.3(26.0)$ & $53.6(25.6)$ & $35.6(24.5)$ & $<0.001$ \\
\hline NIHSS, median \{range\} & $4\{0-40\}$ & $7\{0-40\}$ & $4\{0-40\}$ & 0.315 \\
\hline Prior stroke, any type, No. [\%] & 27 [23.9] & $11[36.7]$ & 16 [19.3] & 0.056 \\
\hline High-risk CES, No. [\%] & $7[6.2]$ & $2[6.7]$ & $5[6.0]$ & 0.999 \\
\hline Lobar ICH, No. [\%] & $22[23.4]$ & $6[26.1]$ & $16[33.5]$ & 0.727 \\
\hline ICH volume, median $\mathrm{ml}\{\mathrm{IQR}\}^{\mathbf{a}}$ & $12\{0.4-97.6\}$ & $12.7\{0.8-97.6\}$ & $11.2\{0.4-87.4\}$ & 0.667 \\
\hline IVH, No. [\%] ${ }^{\mathbf{a}}$ & $36[38.3]$ & $9[39.1]$ & $27[38.0]$ & 0.925 \\
\hline Microbleeds, No. [\%] ${ }^{\mathbf{a}}$ & $51[55.4]$ & $18[81.8]$ & $33[47.1]$ & 0.004 \\
\hline Chronic hematomas, No. [\%] & $30[32.3]$ & $12[52.2]$ & 18 [25.7] & 0.019 \\
\hline WMD score, median $\{$ range $\}$ & $6\{0-12\}$ & $8\{3-12\}$ & $6\{0-12\}$ & 0.004 \\
\hline Baseline DWI lesions, No. [\%] ${ }^{\mathbf{a}}$ & 30 [31.9] & $14[60.9]$ & $16[22.5]$ & 0.001 \\
\hline
\end{tabular}

included larger hematoma volume, intraventricular hemorrhage, microbleeds, and importantly, large reductions in mean arterial BP. A multivariate model predicting ischemic lesions at 1 month included presence of DWI lesions at the baseline time point, prior stroke, microbleeds, and black race.

\section{Prior Literature}

One prior study with a similar demographic profile to that of our cohort evaluated 129 patients with MRI in the first 28 days after acute primary ICH. ${ }^{3}$ DWI abnormalities were identified in $22.9 \%$ of patients and were associated with prior stroke, mean arterial BP lowering, and craniotomy. An analysis of microbleeds was not reported in this study. In a separate study of patients with cerebral amyloid angiopathy, $15 \%$ of patients demonstrated subacute cerebral infarction on DWI studies. ${ }^{4}$ In this cohort, DWI lesions were not associated with recent symptomatic $\mathrm{ICH}$, but were associated with higher microbleed burden.

\section{Mechanisms and Pathophysiology}

The etiology and underlying pathophysiology leading to ischemic lesions remote from the index hematoma are not yet well understood. Putative mechanisms include (1) an active cerebral vasculopathy leading to contemporaneous vessel rupture and occlusion, (2) overly aggressive BP

TABLE 4: Multivariate Logistic Model for Presence of 1-Month DWI Lesions

\begin{tabular}{|lllll|} 
Factor & Odds Ratio & \multicolumn{2}{c}{ 95\% CI } & p \\
Any prior stroke & 4.58 & 1.40 & 14.93 & 0.012 \\
Baseline microbleeds & 3.92 & 1.07 & 14.38 & 0.040 \\
Baseline DWI lesions & 4.72 & 1.53 & 14.63 & 0.007 \\
Black vs nonblack race & 1.37 & 0.36 & 5.18 & 0.641 \\
CI = confidence interval; DWI = diffusion-weighted imaging. & & & \\
\hline
\end{tabular}


lowering in the acute (and possibly subacute) phase in the setting of a diffuse angiopathy, and/or (3) an acute prothrombotic/proinflammatory cascade triggered by the initial ICH or the underlying vasculopathic process itself. Less likely causes include misidentification of hemorrhagic conversion of embolic ischemic lesions (ie, misdiagnosis of primary $\mathrm{ICH}$ ), iatrogenically induced lesions related to diagnostic angiography, or active vasculitis.

Our results strongly suggest an association between DWI lesions in ICH and BP lowering during the acute phase of ICH hospital stay. Historically, the foci of prior studies of BP management in acute $\mathrm{ICH}$ have varied based on the understanding of the underlying pathophysiology of ICH at the time. Initial theories regarding a penumbra of perihematomal ischemia fostered a concern for BP lowering and hypoperfusion in the acute phase. This topic has been controversial; some studies have suggested a transient zone of true ischemia in a subset of patients, whereas others have not. ${ }^{9-11}$ Some advanced imaging studies have additionally identified a perihematomal penumbra of nonischemic, metabolically compromised tissue. ${ }^{12}$ Most recent studies have focused upon the association between early hematoma expansion, elevated BP, and worsened clinical outcome. Two clinical trials have demonstrated the relative safety of early BP lowering with avoidance of substantial neurological deterioration and adverse events, along with modest reductions in hematoma expansion. ${ }^{13,14}$ However, neither of these studies integrated MRI assessments that might identify clinically silent ischemia or microvascular damage.

BP lowering alone, however, may not be sufficient to explain remote ischemia in the setting of acute ICH. We postulate that an interaction may exist between BP lowering and a severe underlying vasculopathy in the setting of $\mathrm{ICH}$. Results from our study and others suggest that imaging and clinical markers of an underlying severe vasculopathy (eg, prior stroke and microbleeds) are independent predictors of ischemic lesions. ${ }^{3,4}$ Disease of the microvasculature, whether due to hypertension or cerebral amyloid angiopathy, has the potential to simultaneously lead to both ischemic and hemorrhagic insults sharing a common underlying microangiopathic process. We hypothesize that the development of ischemic lesions in acute and/or subacute hypertensive ICH is caused by hemodynamic insufficiency due to BP lowering in the setting of a diseased microvasculature that is unable to compensate due to autoregulatory failure. However, it is important to note that our data only demonstrate an association between BP lowering and DWI lesion presence, and further prospective studies are needed not only to confirm this hypothesis, but also to demonstrate that less aggressive BP treatment decreases the rate of lesion development.

\section{Significance}

An understanding of the underlying mechanism(s) and pathophysiology of ischemic lesions in the setting of ICH has important implications for both prognosis and treatment. Our study uniquely characterizes the frequency of DWI lesions at 2 discrete time points (baseline and 1 month). The presence of new ischemic lesions as late as 1 month from ictus demonstrates the active nature of this process. Although the delta MAP did not enter into the 1-month predictive model, this was likely due to colinearity with baseline DWI lesions, which in turn were associated with the delta MAP. This finding suggests that overaggressive BP reductions in the subacute phase of ICH may have important implications. This population of hypertensive patients, often with severe microvascular disease, may have persistent loss of autoregulation. Therefore, BP control within the normal range may not be tolerated, and instead could induce hemodynamic ischemia at the microvascular level.

Moreover, if BP lowering is the predominant factor at either time point, it would be important to understand the clinical significance of the DWI lesions (eg, whether they are clinically silent or have a negative impact on long-term functional outcome or cognitive status). If BP control is associated with poorer outcome, future studies exploring optimal BP control are needed and should include MRI as an outcome marker. However, if ischemic lesions are thrombotic in nature and triggered by the acute ICH and/or caused by an ongoing, active vasculopathy at 1 month independent of BP, future studies would be needed to explore the clinical significance of the lesions, as well as optimal approaches to secondary prevention (eg, role of antiplatelet agents). Our planned analysis of this population with a 1-year MRI will allow us to characterize the frequency of DWI ischemic lesions at a time point far removed from the acute hemorrhage (eg, chronic rate of lesion development in this hypertensive ICH population).

\section{Limitations}

This study had some limitations. There is likely a selection bias toward milder hemorrhages with smaller volumes, as patients with more severe hemorrhages may have been too unstable for MRI. The sensitivity for detection of microbleeds and ischemic DWI lesions may depend on magnet strength. One prior report suggests that $1.5 \mathrm{~T}$ magnets may be more sensitive for detecting hyperacute ischemia on diffusion-weighted imaging. ${ }^{15}$ Because the 1-month scans were preferentially acquired on a $3.0 \mathrm{~T}$ scanner, this may have decreased the overall detection rate at this time point. We did not formally analyze ischemic lesions based upon classical definitions of 
watershed territories. However, it is unclear that diffuse microvascular disease would have a watershed pattern similar to large vessel disease. Finally, we did not routinely collect MR perfusion studies as part of our protocol. Future studies are needed to examine these issues.

\section{Summary and Conclusions}

In conclusion, we found that $>1 / 3$ of patients with $\mathrm{ICH}$ have active cerebral ischemia topographically remote from the acute hematoma at baseline, and importantly, $>1 / 4$ of patients have imaging confirmation of active ischemia in the subacute phase of ICH. Multivariate analyses implicate BP reductions in the setting of an active vasculopathy as an underlying mechanism. Further studies are needed to determine the impact of these lesions on outcome and optimal management strategies to arrest vascular damage.

\section{Acknowledgments}

The project was supported by the National Institute of Neurological Disorders and Stroke, and National Institute on Minority Health and Health Disparities (U54NS057405). The content is solely the responsibility of the authors and does not necessarily represent the official views of the NINDS, the NIMHD, or the NIH.

We thank Dr Dennis Landis for his thoughtful contributions to the article.

\section{Authorship}

R.S.M. was responsible for acquisition of data, study concept or design, analysis or interpretation of data, statistical analysis, and drafting/revising the manuscript for content. R.E.B. was responsible for acquisition of data, analysis or interpretation of data, and drafting/revising the manuscript for content. J.J.W. was responsible for analysis or interpretation of data, statistical analysis, and drafting/ revising the manuscript for content. M.C.G. was responsible for analysis or interpretation of data and drafting/ revising the manuscript for content. N.M.S. was responsible for analysis or interpretation of data and drafting/ revising the manuscript for content. S.F. was responsible for analysis or interpretation of data, statistical analysis, and drafting/revising the manuscript for content. A.J.-T. was responsible for acquisition of data, analysis or interpretation of data, and drafting/revising the manuscript for content. L.G. was responsible for acquisition of data and drafting/revising the manuscript for content. I.S. was responsible for acquisition of data and drafting/revising the manuscript for content. D.E. was responsible for acquisition of data, analysis or interpretation of data, and drafting/ revising the manuscript for content. C.S.K. was responsible for acquisition of data, study concept or design, analysis or interpretation of data, statistical analysis, study supervision or coordination, and drafting/revising the manuscript for content.

\section{Potential Conflicts of Interest}

R.S.M.: speaking fees, Georgetown University; travel expenses, NIH/NINDS. M.C.G.: speaking fees, Novartis Pharmaceuticals. C.S.K.: consultancy, Simcere of America Inc.

\section{References}

1. Hill MD, Silver FL, Austin PC, Tu JV. Rate of stroke recurrence in patients with primary intracerebral hemorrhage. Stroke 2000;31: 123-127.

2. Wijman CA, Venkatasubramanian C, Bruins $S$, et al. Utility of early MRI in the diagnosis and management of acute spontaneous intracerebral hemorrhage. Cerebrovasc Dis 2010;30:456-463.

3. Prabhakaran S, Gupta R, Ouyang B, et al. Acute brain infarcts after spontaneous intracerebral hemorrhage: a diffusion-weighted imaging study. Stroke 2010;41:89-94.

4. Kimberly WT, Gilson A, Rost NS, et al. Silent ischemic infarcts are associated with hemorrhage burden in cerebral amyloid angiopathy. Neurology 2009;72:1230-1235.

5. Menon RS, Kidwell CS. Neuroimaging demonstration of evolving small vessel ischemic injury in cerebral amyloid angiopathy. Stroke 2009;40:e675-e677.

6. Greenberg SM, Vernooij MW, Cordonnier C, et al. Cerebral microbleeds: a guide to detection and interpretation. Lancet Neurol 2009;8:165-174.

7. Fazekas F, Barkhof F, Wahlund LO, et al. CT and MRI rating of white matter lesions. Cerebrovasc Dis 2002;13(suppl 2):31-36.

8. Goldstein LB, Bertels C, Davis JN. Interrater reliability of the NIH stroke scale. Arch Neurol 1989;46:660-662.

9. Rosand J, Eskey C, Chang Y, et al. Dynamic single-section CT demonstrates reduced cerebral blood flow in acute intracerebral hemorrhage. Cerebrovasc Dis 2002;14:214-220.

10. Siddique MS, Fernandes HM, Wooldridge TD, et al. Reversible ischemia around intracerebral hemorrhage: a single-photon emission computerized tomography study. J Neurosurg 2002;96:736-741.

11. Kidwell CS, Saver JL, Mattiello J, et al. Diffusion-perfusion MR evaluation of perihematomal injury in hyperacute intracerebral hemorrhage. Neurology 2001;57:1611-1617.

12. Zazulia AR, Videen TO, Powers WJ. Transient focal increase in perihematomal glucose metabolism after acute human intracerebral hemorrhage. Stroke 2009;40:1638-1643.

13. Antihypertensive treatment of acute cerebral hemorrhage. Crit Care Med 2010;38:637-648.

14. Anderson CS, Huang $Y$, Wang JG, et al. Intensive blood pressure reduction in acute cerebral haemorrhage trial (INTERACT): a randomised pilot trial. Lancet Neurol 2008;7:391-399.

15. Rosso C, Drier A, Lacroix D, et al. Diffusion-weighted MRI in acute stroke within the first 6 hours: 1.5 or 3.0 Tesla? Neurology 2010; 74:1946-1953. 